P93 (continued)

canned beans (refried) than Hispanics. More Hispanics picked corn tortillas (flour), 100\% juice (fruit nectar), and chicken breast (leg) than non-Hispanics.

Conclusions and Implications: These results indicate consumers define healthy foods by the nutritional components; calories, fat, sugar, and salt. Differences in knowledge of nutritious vs. less nutritious food products vary across ethnic identities. Baseline knowledge should be assessed.

Funding: None

\section{P94 Demographics, Anthropometric Measurements, Vegetable Consumption, and Food Waste of Community Supported Agriculture (CSA) Members in Central Minnesota}

Emily Heying, PhD, emily.heying@gmail.com, St. John's University, 37 College Avenue South, Saint Joseph, MN 56374; Kendra Butkowski, BA, St. John's University

Objective: To investigate the relationship between demographics/anthropometric profiles and vegetable consumption and food waste in central Minnesota Community Supported Agriculture (CSA) members.

Study Design, Setting, Participants: After IRB approval, participants were recruited from the CSA member pool prior to the 2016 CSA season. Participants chose a 4-digit ID number to ensure confidentiality and allow for comparison of data over time. Participants were given an initial survey inquiring about demographics, anthropometric profiles and then were surveyed for four weeks regarding their vegetable consumption and food waste from the CSA. A final survey inquired about self-efficacy regarding eating and waste behavior.

Outcome Measures and Analysis: After the CSA season ended, the weekly survey data were pooled and compared. An ANOVA test was utilized to determine differences in food waste and vegetable consumption among demographics/anthropometrics such as income level, ethnicity, and BMI.

Results: Demographics for participants and spouses were as follows $(n=36)$ : 94\% white/Caucasian, 64\% had earned a master's degree or higher, and $47 \%$ had a combined household income of $>\$ 100,000$. The average BMI for women and men were $25.7+4.5$ and $25.7+5.5 \mathrm{~kg} / \mathrm{m} 2$, respectively. Vegetable consumption and food waste did not differ based on demographics or anthropometrics. No correlations between self-efficacy of eating or waste behaviors and actual vegetable consumption and food waste were detected.

Conclusions and Implications: CSA shares increase vegetable consumption during the season. However, current research indicates that those who have access to CSA shares are predominately white/Caucasian, welleducated, and have an income well above poverty. The results of this study confirm existing research and indicate the need to explore avenues to expand CSA accessibility to under resourced populations.

Funding: CSB/SJU Undergraduate Research Grant, CSB Nutrition Department

\section{P95 Elementary School Gardens: Survey Findings Identifying Barriers and Opportunities for Garden-Based Learning}

Diane Smith, MA, RD, diane.smith@wsu.edu, WSU Extension Skagit County, 11768 Westar Lane, Burlington, WA 98233; Christina Hansen, MS, Anacortes School District; Tessa Bryant, BA, WSU Americorps

Objective: A survey of elementary schools in Skagit County, WA identified barriers and opportunities to having a garden on school grounds and describes how the school gardens are used in instruction such as science, health, and food systems.

Rationale: School garden programs are gaining popularity in elementary schools in the United States evidenced by a substantial increase from $11.4 \%$ in 2006 07 to $26.6 \%$ in the 2012-13 school year (Turner, 2014). Encouraged by Michelle Obama's establishment of the White House garden in concert with celebrity food education activists like Alice Waters and Jamie Oliver there is increasing attention on getting American school children outside and growing food.

Description: Target audience was Community Educators, Extension, Curriculum Specialists. Fourty-four perecent of elementary schools in Skagit County reported having a school garden used in school instruction and after-school programming. A survey was conducted to describe the status of school gardens and the garden-based curricula in use. The study explored if, and if so how, school gardens influenced fruit and vegetables intake to address the overweight epidemic, impact food access and increase learning opportunities.

Evaluation: Common barriers for the successful management and coordination of elementary school gardens were consistent across both schools with and without gardens. Garden organization, budget, structure, and sustainability were key challenges experienced. Evidence-based curriculum consistent with Common Core learning objectives was limited and teachers did not have enough time nor receive training to include garen-based learning in lesson plans.

Implications: There is interest in utilizing gardens as an experiential learning strategy; however, low utilization of garden space for intentional garden-based learning was evident in survey responses. A need for organizational support, evidence-based curriculum, and teacher training is indicated. Funding: None

\section{P96 Evaluating Barriers to SNAP Acceptance in Alabama Farmers Markets}

Krysta P. Kellegrew, BS, RD, Alabama Cooperative Extension System/Auburn University; Alicia R. Powers,PhD, arp0042@auburn.edu, Alabama Cooperative Extension System/Auburn University, 206 Duncan Hall, Auburn University, AL 36849;

Barb Struempler, PhD, Alabama Cooperative Extension System/Auburn University; Sondra Parmer, PhD; Katie Funderburk, RD, MS 
P96 (continued)

Objective: Farmers markets can increase access to healthy food options, especially in low-income communities where fruits and vegetables are not otherwise available. The Supplemental Nutrition Assistance Program (SNAP) acts as a catalyst to increase availability and appeal of fresh fruits and vegetables in low-income communities by overcoming cost barriers of these foods in various settings, including farmers markets. In 2015, only 38\% of farmers markets nationwide accepted SNAP benefits. The purpose of this non-experimental study is to determine barriers perceived by Alabama farmers related to accepting SNAP at farmers markets.

Study Design, Setting, Participants, Intervention: This study utilizes the Social Ecological Model constructs to describe socio-demographic characteristics, perceived barriers and potential solutions to accepting SNAP at farmers markets.

Outcome Measures and Analysis: On average, 10 farmers participated in six regional, pre-season grower meetings sponsored by the Alabama Farmers Market Authority in early 2017 where researchers facilitated completion of a 51-question, anonymous survey.

Results: Farmers identified distinct barriers to accepting SNAP at farmers markets: limited information about the application process, complexity of the application process, limited information about the payment system and time and resources necessary to use the payment system. Farmers expressed a need for greater support in promoting SNAP redemption at farmers markets.

Conclusions and Implications: Understanding key barriers and potential solutions for SNAP acceptance and redemption in farmers markets is vital for successful utilization of SNAP. Findings from this study will guide development of strategies and resources aimed at increasing the number of farmers accepting SNAP at Alabama farmers markets, ultimately increasing access to fresh fruits and vegetables in low-income communities.

Funding: Supplemental Nutrition Assistance Program Education

\section{P97 WITHDRAWN}

\section{P98 Behavioral Changes and Learning Differences in Students Registered in Online Versus In-Seat General Education Nutrition Classes}

AnnaCahn, MS, RD, acahn27@gmail.com, California State University, Long Beach, 1250 Bellflower Boulevard, Long Beach, CA 90840

Objective: The purpose of this thesis was to investigate knowledge, motivational and behavioral changes among students enrolled in an in-seat, face-to-face introductory nutrition course compared to students enrolled in the same course online.

Theory, Prior Research, Rationale: Colleges and universities across the country are adapting to the increased demand for higher education and are looking for alternative ways for students to receive credits. This includes providing different modalities of learning, including online, hybrid, and in-seat classes.

Description: The target audience for this thesis is for college and university nutrition professors and course developers. A twenty-nine question pre- and post-semester survey consisting of questions related to nutrition knowledge, motivation, and behavior was distributed electronically to 228 students enrolled in selected in-seat and online sections of an introductory nutrition course offered at California State University, Long Beach. Differences between groups (in-seat vs. online) were investigated using independent samples t-tests.

Evaluation: Of the 170 students who participated, there were no significant differences in knowledge, motivation, and behavior between the in-seat and online students at 